\title{
Bilateral Isolated Spherophakia in Two Young East European Siblings: A Case Report
}

\author{
Silvia Babighian Silvia Bini Alessandro Galan \\ Department of Ophthalmology, Azienda Ospedaliero-Universitaria of Padova, San Antonio \\ Hospital, Padova, Italy
}

\section{Keywords}

Angle closure glaucoma · Case report · Crystalline lens dislocation · Spherophakia

\begin{abstract}
A 19-year-old Caucasian woman was referred to the emergency room and thereafter to the department of ophthalmology complaining for bilateral decrease of visual acuity and severe pain. A complete ophthalmological evaluation was performed. Best-corrected visual acuity (BCVA) was LogMAR 0.3 in the right eye (RE) and LogMAR 0.5 in the left eye (LE). Intraocular pressure (IOP) was 28 and $38 \mathrm{~mm} \mathrm{Hg}$ in the RE and LE, respectively. The patient showed a shallow anterior chamber and spherical equivalent refractive error -29.0 diopters (D) in the $\mathrm{RE}$ and $-30.0 \mathrm{D}$ in the LE. The diagnosis of bilateral angle closure glaucoma, secondary to highly myopic, forward dislocated lens was made, in the setting of spherophakia. The ultrasound biomicroscopy images confirmed the diagnosis. Clear lens extraction was promptly performed with resolution of ocular hypertension and restoration of BCVA. In view of the frequent systemic association, family members also underwent ophthalmological evaluation. The 13-year-old sibling showed mild myopia and borderline IOP. He was administered topical $\beta$-blockers and observation. Genetic counseling did not reveal mutations usually associated with spherophakia or systemic conditions. This case report highlights the variable spectrum of clinical expression in spherophakia; therefore, ophthalmological treatment should be tailored according to clinical presentation. Systemic evaluation and genetic counseling are also recommended in the suspicion of spherophakia.
\end{abstract}


Babighian et al.: Isolated Spherophakia in Two Siblings

\section{Introduction}

Spherophakia is a rare eye disease that may present as an isolated condition or, more frequently, it can be associated to other clinical features, in the setting of a systemic syndrome [1]. Whether the presentation is isolated or in the contest of a syndrome, spherophakia is constantly characterized by developmentally hypoplastic lens zonules which are abnormally weak [2]. For this reason, crystalline lens changes its normal shape to spherical, with increased anteroposterior thickness and reduced equatorial diameter. This condition is therefore associated with varying degrees of lenticular myopia, even unacceptably elevated [1]. Hypoplastic zonular fibers may also be responsible for crystalline lens subluxation or dislocation, leading, in some cases, to pupillary-block glaucoma [1-4].

Spherophakia can be associated with systemic syndromes. Some of the most common associations are Marfan's and Weill-Marchesani's syndromes, and mandibulofacial dysostosis, Alport's, Klinefelter's, Sturge Weber, and Axenfield-Rieger syndromes [1-7]. Spherophakia could also be associated with diseases secondary to abnormal development of the eye and specifically altered maturation of the anterior segment, such as irido-corneal endothelial syndrome, megalocornea, and aniridia [7]. Isolated or unilateral spherophakia is rare; rather, it is typically bilateral and commonly associated with genetic disorders [1-5]. It has also been frequently described in consanguinity [8]. The aim of this report was to describe the clinical features and management of 2 young East-European siblings, affected by bilateral spherophakia.

\section{Case Presentation}

A 19-year old Caucasian woman (PT1) was admitted to the emergency room and ophthalmological department complaining for rapid bilateral visual acuity decrease and severe headache, unresponsive to pharmacological treatment. A complete ophthalmological examination was performed, including best-corrected visual acuity (BCVA) evaluation, slit lamp biomicroscopy, intraocular pressure (IOP) measurement, and fundus examination. Anterior segment photography (Digital LED Slit lamp led, CSO Ophtalmic-SL9900) and high-frequency ultrasound biomicroscopy (UBM, resolution $50 \mathrm{MHz}$ ) were also performed.

At first ophthalmological evaluation, PT1 revealed BCVA = LogMAR 0.3 (Snellen 20/40) in the right eye (RE) and LogMAR 0.5 (Snellen 20/63) in the left eye (LE). Spherical equivalent refractive error was -29.0 diopters (D) and $-30.0 \mathrm{D}$ in the RE and LE, respectively. Slit lamp examination revealed in both eyes the presence of a shallow anterior chamber (Fig. 1a-b), and gonioscopy confirmed the presence of extremely narrow angle (Shaffer grade 1). The IOP measurement with Goldmann applanation tonometry was 28 and $38 \mathrm{~mm} \mathrm{Hg}$ in the RE and LE, respectively. Pharmacological pupil dilation was obtained with cyclopentolate eye drops. Mydriatic observation of the anterior segment showed subluxated crystalline lens in both eyes (Fig. 1a-b). Pupils' dilation yielded to significant reduction of IOP, to normal value in both eyes (15 mm Hg). Fundus examination was unremarkable in both eyes. On A-scan biometry, the axial length of PT1 was 23.95 and $23.22 \mathrm{~mm}$ in the RE and LE, respectively. Lens thickness (anteroposterior diameter) of PT1 was 4.54 and $4.30 \mathrm{~mm}$ in the RE and LE, respectively, measured with UBM in the cycloplegic condition. Anterior chamber depth (ACD) was reduced to $0.5 \mathrm{~mm}$ in both eyes. The corneal diameter was $12 \mathrm{~mm}$ in both eyes (normal diameter excluding megalocornea), and keratometric values were $42.07 \mathrm{D} / 43.09 \mathrm{D}\left(176^{\circ} / 86^{\circ}\right)$ in the RE and $42.47 \mathrm{D} / 43.00 \mathrm{D}\left(17^{\circ} / 107^{\circ}\right)$ in the LE. UBM confirmed the diagnosis of spherophakia associated with a pupillary block glaucoma, secondary to forward-dislocated lens (Fig. 1c). A severe zonular laxity due to weak zonule fibers was also detected.

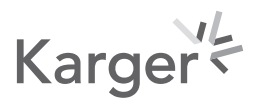


Fig. 1. a Slit lamp photography of anterior segment of the RE of a young patient (named PT1 in the main text) affected by spherophakia and angle closure glaucoma. b Note the crystalline lens in the retro-illuminated image. c UBM image. RE, right eye; UBM, ultrasound biomicroscopy.

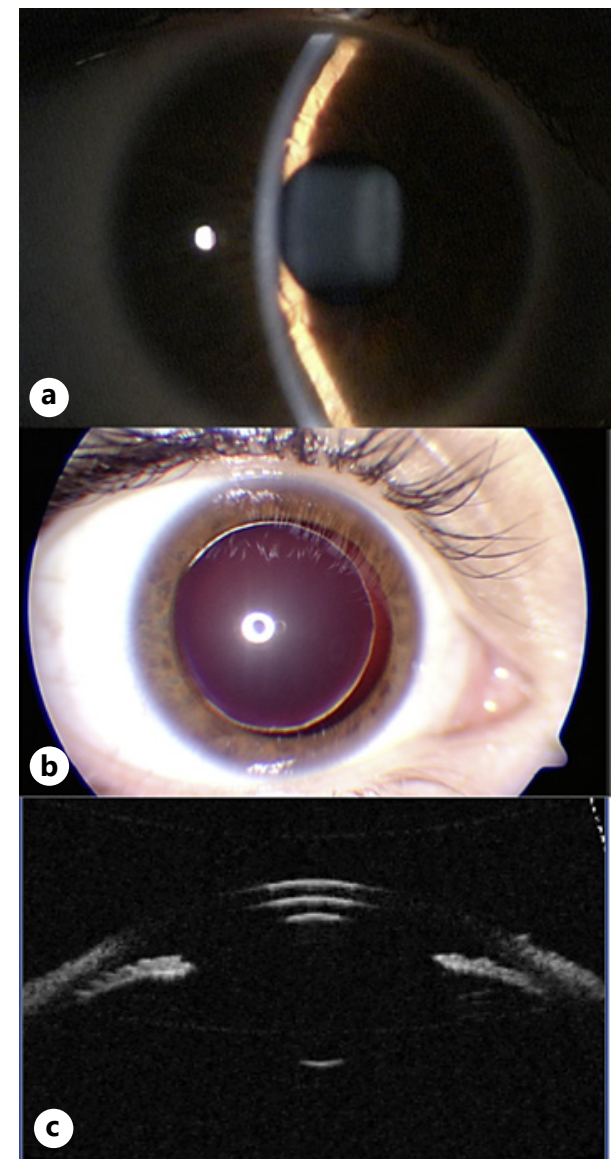

After clinical and instrumental evaluation, the diagnosis of bilateral angle closure glaucoma secondary to spherophakia was made. PT1 underwent prompt ocular surgery. An expert surgeon (A.G.) performed clear lens extraction by phacoemulsification without intraocular lens (IOL) implantation, in both eyes [9]. Ocular surgery was successfully performed under local peri-bulbar anesthesia in both eyes, and no intraoperative or postoperative complications occurred. After lensectomy, the surgeon did not implant the IOL because of severe instability of the capsular bag evaluated intraoperatively and to avoid IOL contact with corneal endothelium. Anterior vitrectomy was not necessary. Postsurgical refractive error was corrected by means of customized contact lenses. Postoperatively, IOP was successfully controlled with $\beta$-blocker eye drops (timolol 0.5\%) twice a day. At 1 month follow-up, BCVA improved to LogMAR 0.0 (Snellen 20/20), and ACD was restored at slit lamp examination (Fig. 2).

Spherophakia frequently runs in families; therefore, family members underwent ophthalmological screening. Among family members, ophthalmological evaluation was suggestive of bilateral spherophakia in the younger (13-year-old) male sibling (PT2), despite the presence of mild clinical features compared to PT1. Patient's parents were not available for ophthalmological evaluation.

PT2 was asymptomatic. Ophthalmological examination of PT2 revealed myopia - 6 D and IOP within a normal range, despite a borderline value $(21 \mathrm{~mm} \mathrm{Hg})$. The equator of crystalline lens was visible at full mydriasis, the anteroposterior diameter of the lens was $3.8 \mathrm{~mm}$ in both eyes, with axial length 23.12 in the RE and 23.43 in the LE. Keratometric values of corneal curvature were $44.56 \mathrm{D} / 45.86 \mathrm{D}\left(179^{\circ} / 89^{\circ}\right)$ in the $\mathrm{RE}$ and $44.78 \mathrm{D} / 45.85 \mathrm{D}\left(4^{\circ} / 94^{\circ}\right)$ in the 


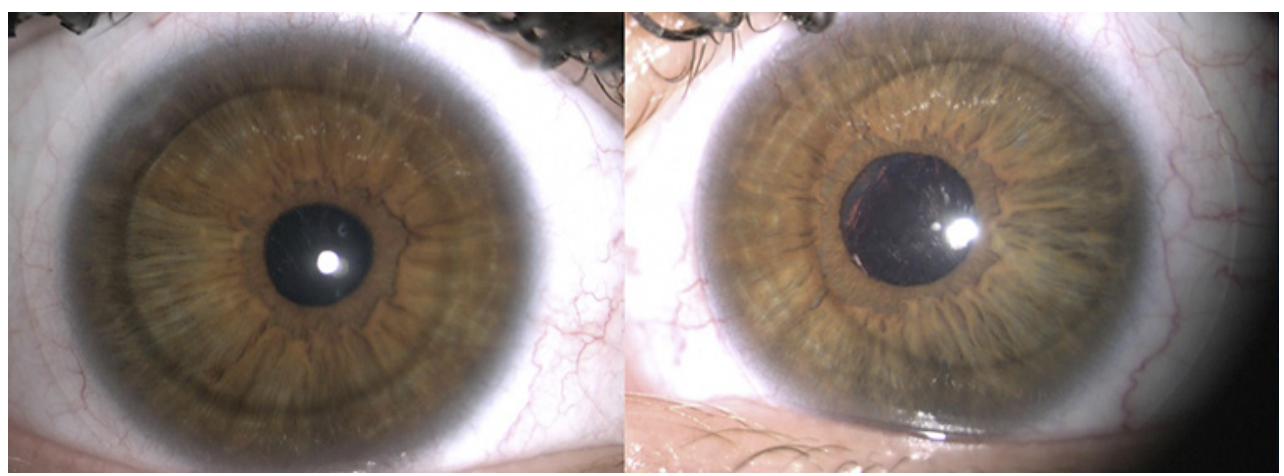

Fig. 2. Slit lamp photography of anterior segment of the RE (a) and LE (b) of a young patient affected by spherophakia and angle closure glaucoma, after clear lens extraction without IOL placement. Note the presence of contact lenses for visual rehabilitation. RE, right eye; LE, left eye; IOL, intraocular lens.

LE. Corneal diameter was 12.5 in both eyes (normal diameter excluding megalocornea). Anterior chamber angle was open (Shaffer grade 2-3). An UBM-expert ophthalmologist (S.B.) examined PT2: biomicroscopy revealed a severe-to-moderate reduction of ACD $=2.1 \mathrm{~mm}$ and $1.9 \mathrm{~mm}$ in the RE and LE, respectively. Based on these clinical findings, PT2 underwent observation; however, topical treatment with beta-blocker eye drops (timolol $0.5 \%$ ) was started to prevent undetected IOP spikes.

At the last follow-up visit in October 2020, PT1 was clinically stable, and treatment was well tolerated. The measurement of crystalline lens anteroposterior diameter of PT2 showed an increased thickness (4.38 in the RE and 4.30 in the LE); however, the patient was still asymptomatic with tolerable progression of myopia.

Family history revealed consanguineous relatives. General health examination of both siblings was unremarkable. Genetic testing for systemic associations was performed in both PT1 and PT2, and did not reveal any significant mutation. Mutations in ADAMTS family and FBN-1 genes are typically associated with micro-spherophakia in the setting of systemic syndromes such as Weill Marchesani and Marfan's syndrome. However, new findings of sporadic cases of micro-spherophakia have been recently detected. LTBP2 gene, for example, has been found in a patient with spherophakia and consanguinity [5]. However, in this case, the genetic test was inconclusive.

Informed consent was obtained for both patients, and anonymized clinical information was collected, according to general data protection regulation (EU-2016/679). In accordance with local guidelines, as specifically stated by the Ethics Committee for Clinical Practice of Azienda Ospedaliero-Universitaria di Padova, ethics approval was not required for this study. The study was conducted in accordance with the Declaration of Helsinki.

\section{Discussion}

Spherophakia is a rare eye condition frequently associated with systemic disorders, such as Weill-Marchesani's, Marfan's, Klinefelter's, or Alport's syndromes [1-4]. New associations are still emerging in recent reports, such as with Sturge Weber's syndrome [6]. Although the pathophysiology of spherophakia is not completely clarified yet, it is known that zonular fibers' weakness is responsible for crystalline lens assuming spherical shape. Fibers' weakness combined to the high elasticity of crystalline lens in young patients leads the lens to assume its natural spherical shape [10]. The increased lens focusing power induces a variable degree

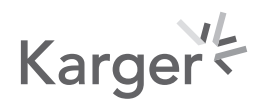


of myopia (even elevated $>20 \mathrm{D}$ ). In a small number of cases, pupillary block glaucoma may occur, due to forward displacement of the lens. The therapeutic approach is debated and must be tailored on each specific case. Lensectomy has been considered an option for managing the dislocated lens and control glaucoma $[1,11]$. Visual rehabilitation after lensectomy may be obtained with aphakic spectacles, contact lenses, or with IOL implant [1]. When IOL implant is planned, iris-enclavated IOLs are common choices, and posterior chamber and scleralfixated IOL have also been described [1]. Capsular tension ring has been considered in the literature; however, in spherophakia, the reduced diameter of the capsular bag, zonular weakness, patient's age, and surgeon's experience should be carefully considered. In this case of spherophakia in a young woman, the use of tensional ring and IOL implant were considered inappropriate. The use of a tension ring (or even Cionni ring), in fact, could induce complications in this eye with marked zonular weakness. The eventual need of surgical ring removal and repeated ocular surgery in a young patient were not justified by clinical advantages in this case.

Our patients (PT1 and PT2) had no clinical features suggestive of any associated systemic condition; therefore, we did not expect the presence of mutations in genes such as ADAMTS family or FBN-1. The genetic test did not reveal any significant mutation either in other genes. Although a new mutation in a case of consanguinity could be expected, inconclusive genetic results were found in our case.

To our knowledge, this report represents an original case of spherophakia in young, healthy consanguineous siblings, presenting in PT1 with bilateral simultaneous acute angle closure glaucoma and in PT2 with myopia and borderline ocular tension. Clear lens extraction without IOL implantation was considered the primary therapeutic modality for spherophakia associated to angle closure glaucoma in PT1. Although posterior vitreous pressure was expectable, anterior vitrectomy and intravenous mannitol use were not necessary (mannitol use, by dehydration of vitreous body, may also elicit choroidal detachment). This might be explained because of marked posterior shift of the irido-lenticular diaphragm when the patient was held is the supine position, creating adequate ACD for lensectomy. Moreover, the skills and experience of the surgeon were crucial for the safe execution of the surgical procedure. The severe zonular laxity and reduced dimensions of the capsular bag led to evaluation of inappropriate IOL implant in this case. Visual rehabilitation was successfully obtained with contact lenses, showing BCVA = LogMAR 0.0. The excellent refractive result obtained with contact lenses after lensectomy, and their good tolerance to date, once again led to postpone the option of IOL implant, and PT1 is still aphakic to date. However, the possibility of a delayed IOL implantation could be considered during future follow-up. A nonsurgical approach was planned for PT2. Both patients are under lifetime ophthalmological follow-up so that long-term postoperative course of PT1 and clinical course changes of PT2 can be monitored.

In conclusion, this case report highlights that spherophakia may have a different spectrum of ocular manifestations. In the suspicion of spherophakia, a careful ophthalmological evaluation including UBM is strongly recommended, including a family members' screening. Moreover, a multidisciplinary evaluation with genetic counseling is appropriate, in the attempt to detect even new unknown sporadic mutations. The ophthalmologists should therefore recommend ophthalmological evaluation to all family members, in order to detect affected eyes even before the appearance of clinical manifestation, and plan a tailored treatment (or monitoring), according to each clinical presentation.

\section{Acknowledgment}

This manuscript does not include any nonauthor contributors to acknowledge.

\section{Karger'}


Babighian et al.: Isolated Spherophakia in Two Siblings

\section{Statement of Ethics}

This case report was conducted in accordance to good clinical practice and ethically with the World Medical Association Declaration of Helsinki. Written informed consent was obtained from participants for publication of the details of their medical case and any accompanying images. This study protocol was reviewed, and the need for approval was waived by the Ethics Committee for Clinical Practice (CESC) of AOP (Azienda Ospedaliera di Padova). Ethics approval was not required for this study.

\section{Conflict of Interest Statement}

The authors have no financial disclosures and no commercial or proprietary interest in any materials discussed in this report.

\section{Funding Sources}

This manuscript did not receive any funding.

\section{Author Contributions}

Silvia Babighian and Alessandro Galan conceived the manuscript. Silvia Babighian collected all clinical data. Silvia Babighian and Silvia Bini wrote the draft of the manuscript. All authors have approved the final version of the manuscript.

\section{Data Availability Statement}

All data generated or analyzed during this study are included in this article. Further inquiries can be directed to the corresponding author.

\section{References}

1 Khokhar S, Pillay G, Sen S, Agarwal E. Clinical spectrum and surgical outcomes in spherophakia: a prospective interventional study. Eye. 2018;32:527-36.

2 Macken PL, Pavlin CJ, Tuli R, Trope GE. Ultrasound biomicroscopic features of spherophakia. Aust N Z J Ophthalmol. 1995;23:217-20.

3 Johnson GJ, Bosanquet RC. Spherophakia in a Newfoundland family: 8 years' experience. Can J Ophthalmol. 1983;18:159-64.

4 Pikkel J, Irena E. Isolated spherophakia and glaucoma. Case Rep Med. 2013;2013:516490-3.

5 Desir J, Sznajer Y, Depasse F, Roulez F, Schrooyen M, Meire F, et al. LTBP2 null mutations in an autosomal recessive ocular syndrome with megalocornea, spherophakia, and secondary glaucoma. Europ J Hum Genet. 2010;18:761-7.

6 Avalos-Lara SJ, Antonio-Aguirre B, Perez-Ortiz AC, Mendoza Velásquez C, Camacho-Ordoñez A, Palacio Pastrana C. Spherophakia and ectopia lentis in a Sturge-Weber patient: a case report. Case Rep Ophthalmol. 2020;11:356-63.

7 Mannino G, Abdolrahimzadeh B, Calafiore S, Anselmi G, Mannino C, Lambiase A. A review of the role of ultrasound biomicroscopy in glaucoma associated with rare diseases of the anterior segment. Clin Ophthalmol. 2016;10:1453-9.

8 Ben Yahia S, Ouechtati F, Jelliti B, Nouira S, Chakroun S, Abdelhak S, et al. Clinical and genetic investigation of isolated microspherophakia in a consanguineous Tunisian family. J Hum Genet. 2009;54:550-3. 
9 Richdale KL, Jones LA, Mitchell GL, Zadnik K, Mutti DO, Bullimore MA. Crystalline lens thickness from infancy to adulthood. Invest Ophthalmol Vis Sci. 2008;49:3137.

10 Willoughby CE, Wishart PK. Lensectomy in the management of glaucoma in spherophakia. J Cataract Refract Surg. 2002;28:1061-4.

11 Lo Giudice G, Angelini E, Bini S, Candian T, Crudeli C, Galan A. Outcome of cataract surgery in children affected by malignancies other than retinoblastoma with eye-lens radiation exposure. European J Ophthalmol. 2021. Epub ahead of print. 